\title{
Photometry of Active Centaurs: Colors of Dormant Active Centaur Nuclei
}

\author{
Ian Wong ${ }^{1,4}$ (1) , Aakash Mishra ${ }^{2}$, and Michael E. Brown ${ }^{3}$ (1) \\ ${ }^{1}$ Department of Earth, Atmospheric, and Planetary Sciences, Massachusetts Institute of Technology, Cambridge, MA 02139, USA; iwong@mit.edu \\ ${ }^{2}$ Davis Senior High School, Davis, CA 95616, USA \\ ${ }^{3}$ Division of Geological and Planetary Sciences, California Institute of Technology, Pasadena, CA 91125, USA \\ Received 2019 March 11; revised 2019 April 17; accepted 2019 April 18; published 2019 May 13
}

\begin{abstract}
We present multiband photometric observations of nine Centaurs. Five of the targets are known active Centaurs (167P/CINEOS, 174P/Echeclus, P/2008 CL94, P/2011 S1, and C/2012 Q1), and the other four are inactive Centaurs belonging to the redder of the two known color subpopulations (83982 Crantor, 121725 Aphidas, 250112 2002 KY14, and 2813712008 FC76). We measure the optical colors of eight targets and carry out a search for cometary activity. In addition to the four inactive Centaurs, three of the five active Centaurs showed no signs of activity at the time of observation, yielding the first published color measurements of the bare nuclei of $167 \mathrm{P}$ and P/2008 CL94 without possible coma contamination. Activity was detected on P/2011 S1 and C/2012 Q1, yielding relatively high estimated mass loss rates of $140 \pm 20$ and $250 \pm 40 \mathrm{~kg} \mathrm{~s}^{-1}$, respectively. The colors of the dormant nuclei are consistent with the previously published colors, indicating that any effect of non-geometric scattering from Centaur dust or blanketing debris on the measured colors is minimal. The results of our observations are discussed in the context of the cause of Centaur activity and the color distributions of active and inactive Centaurs. We suggest that the relative paucity of red Centaurs with low-perihelion orbits may not be directly due to the blanketing of the surface by unweathered particulates, but could instead be a result of the higher levels of thermal processing on low-perihelion Centaurs in general.
\end{abstract}

Key words: minor planets, asteroids: general - planets and satellites: surfaces - techniques: photometric

\section{Introduction}

Centaurs are a population of minor bodies located in the middle-outer solar system and are conventionally defined to be objects with orbital semimajor axes and perihelion distances between those of Jupiter and Neptune. These objects originated farther out in the Kuiper Belt and were scattered inward onto unstable orbits with dynamical lifetimes around $10 \mathrm{Myr}$ (Tiscareno \& Malhotra 2003). Their smaller heliocentric distances have made Centaurs ideal proxies for studying the more distant population of Kuiper Belt objects (KBOs), from which they are sourced. More broadly, understanding the composition of Centaurs and KBOs may provide crucial constraints on the properties of the outer regions of the primordial protoplanetary disk, with major implications for models of the formation and evolution of the solar system.

Spectroscopic and photometric observations have revealed that Centaurs are generally characterized by redder-than-solar colors and very low albedos. Most notably, the optical color distribution of Centaurs is strongly bimodal, dividing the population into two groups: the gray and red Centaurs (e.g., Peixinho et al. 2003; Romanishin \& Tegler 2018). Until recently, the origin of this bifurcation in color remained an open question. By analyzing the colors of KBOs, several authors suggested that they are likewise bimodal in color (Fraser \& Brown 2012; Peixinho et al. 2012). However, at the time, well-measured KBO colors were relatively few and largely obtained through targeted observations. As such, the observational biases were difficult to quantify, and our understanding of the underlying color distribution of KBOs was uncertain.

Recent surveys of small KBOs in the same size range as Centaurs $(d<300 \mathrm{~km}$, assuming a visible albedo of $\sim 0.1)$ have

\footnotetext{
${ }^{4} 51$ Pegasi b Postdoctoral Fellow.
}

definitively resolved the question of Centaur color bimodality (e.g., Wong \& Brown 2017; Schwamb et al. 2019). These systematic photometric observations have revealed that all dynamical KBO classes except the cold classicals also display a robust color bimodality. Moreover, the measured color centers of the two color subpopulations are identical to the ones observed in the gray and red Centaurs. This indicates that the observed optical color bimodality among Centaurs is an inherited feature from their source population-the KBOs.

A subset of Centaurs of particular interest is the active Centaurs. These unusual objects have been observed to display comet-like behavior at times, expelling gas and dust to form a dispersed coma of material around a central nucleus. The activity of these Centaurs provides a unique window into their chemical and physical characteristics and may offer crucial insight into the composition and evolutionary history of minor bodies in the outer solar system in general. A notable property of active Centaurs is that their measured color distribution has been shown to differ from that of the overall Centaur population. Specifically, active Centaurs appear to generally lack the very red surface colors found among the red Centaur subpopulation (e.g., Jewitt 2009, 2015).

There are three salient open questions concerning active Centaurs. First, there is the question of whether the activity itself is systematically affecting the measured colors of active Centaurs. The vast majority of the color measurements of active Centaurs in the published literature have been obtained when the bodies were active. Non-geometric scattering off optically small coma particles ejected from an active Centaur could introduce a relatively blue component to the photometry, as has been observed, for example, on some short-period comets (e.g., A'Hearn et al. 1989). The second question is whether both primordially gray and red KBOs can become active when scattered inward onto Centaur orbits. It is possible 
that the cometary activity of these objects could be blanketing the surface with pristine material from below the highly irradiated outer layers (e.g., Delsanti et al. 2004; Jewitt 2009, 2015), which may have a distinct color and thus obscure the original color of the body prior to activity. Alternatively, the apparent color-activity dependence could be reflective of different physical properties, which would suggest that the gray and red Centaurs, and by extension gray and red $\mathrm{KBOs}$, may have formed in separate regions of the protoplanetary disk. This second question has been nominally settled by the recent discovery of the first active red Centaur (Mazzotta Epifani et al. 2018), though the aforementioned discrepancy between the overall color distributions of active and inactive Centaurs remains unaddressed.

Lastly, the causes of Centaur activity and its effects on the surface properties of these objects are not fully understood. The orbital perihelia of active Centaurs are significantly smaller than those of inactive Centaurs (Jewitt 2009), suggesting that the activity is primarily dependent on surface temperature. The heliocentric distances at which active Centaurs have been observed to display comae are consistent with the activity being triggered by the crystallization of amorphous ice and the concomitant release of fine-particle dust and trapped volatile gases, such as carbon monoxide (e.g., Capria et al. 2000; Jewitt 2009; Wierzchos et al. 2017). However, the importance of secondary processes affecting the surface chemistry remains unknown.

As part of the ongoing effort to better understand the nature of active Centaurs, we carried out a photometric study of nine Centaurs. These observations targeted in particular active Centaurs that have passed perihelion and are at larger heliocentric distances than when they were previously observed to be active. The main objective was to look for cessations in activity and obtain color measurements of dormant active Centaur nuclei without the possible contamination from coma material. A complementary goal was to search for the onset of activity on known red Centaurs. Monitoring the activity of Centaurs provides strong constraints on the causes of activity, with major implications for our understanding of the physical properties and chemical composition of these objects.

\section{Observations and Data Analysis}

We carried out photometric observations of Centaurs on three nights in 2017-February 15-16 and August 21. The targets were imaged in three bands $-B(390-490 \mathrm{~nm}), V$ (505-595 nm), and $R(570-690 \mathrm{~nm})$ - using the Large Format Camera (LFC) instrument on the 200 inch Hale Telescope at Palomar Observatory. The science detector in LFC consists of an array of six $2048 \times 4096$ CCDs with a pixel scale of 0 ". 18 . We applied $2 \times 2$ binning to reduce readout time, resulting in an effective pixel scale of 0 ". 363 . Bias frames and dome flats were acquired at the beginning of each night prior to science observations. Exposure times were calculated so as to ensure a per-filter signal-to-noise ratio of at least 50, assuming typical observing conditions at Palomar; imaging in the $R$-band, where Centaurs are intrinsically brighter, was extended to increase sensitivity to activity, yielding signal-to-noise ratios exceeding 100 in many cases.

For all but the brightest targets, observations in each filter were split across multiple exposures. Filters were cycled sequentially to minimize the effect of possible rotational brightness modulation on the color measurements. Several of our targets have published rotational light-curve measurements: all of them have periods longer than $7 \mathrm{hr}$ and peak-to-peak photometric amplitudes of less than $0.25 \mathrm{mag}$, while the overall mean Centaur rotational period from light-curve surveys is around $9 \mathrm{hr}$ (Duffard et al. 2009). For our observations, the maximum time baseline for consecutive $B-V-R$ exposures is under 20 minutes. It follows that the expected contribution of rotational light-curve modulation to individual color measurements is small. This methodology is empirically validated when comparing independent $B-V$ and $V-R$ color measurements that we obtained for the same object-in all cases, the measurements are self-consistent at better than the $1.5 \sigma$ level. Therefore, there is no statistically significant effect of the objects' rotation on our color measurements.

Observations during the two consecutive February nights were plagued by intermittent cloud cover, high sky background, and non-photometric conditions. Only several hours' worth of observation on 2017 February 15 produced data of sufficient quality to yield reliable colors. Conditions on 2017 February 16 were significantly better, and we dedicated that evening's observations to long exposures, primarily in the $R$ band, in search of activity. As a result, several of the Centaurs imaged on the 2017 February nights do not have $B-V$ and $V-R$ color measurements. For the exposures from the February nights that we used in our analysis, the seeing ranged from 1".0 to 2" 0 . Conditions on 2017 August 21 were good, with clear skies throughout the night. Seeing during the final night mostly varied between 1 ". 2 and 1 ". 8 .

The images were run through a pipeline that handles data reduction, image processing, and photometric calibration using standard techniques for photometric observations of moving objects. Following bias-subtraction and flat-fielding, we detected bright sources in each image and automatically matched them with stars in the Pan-STARRS DR1 catalog (Flewelling et al. 2016) to produce an astrometric solution. Next, the magnitudes from the Pan-STARRS catalog were converted to $B, V, R$ magnitudes using the empirical conversions calculated by Tonry et al. (2012), and the photometric zero-point was calculated for each image in the respective band. The instantaneous position of each target at the time of exposure was determined by querying the JPL Horizons database, from which the corresponding source on the image was identified and its apparent magnitude measured. We utilized fixed circular apertures for photometric extraction, ranging in size from 3 to 8 pixels in radius, and chose the optimal aperture radius for each object that minimized the resultant uncertainty in the color measurements. Exposures in which the target was situated in close proximity to other sources or overlapped with hot pixels and/or cosmic rays were removed from our analysis. The details of our observations are listed in Table 1.

To search for Centaur activity, we constructed surface brightness profiles (SBPs) using multi-aperture $R$-band photometry for the target and 3-5 non-saturated bright stars in each image. The flux density within concentric annuli one pixel wide was computed and plotted as a function of distance from the centroid. The SBPs of stellar sources were averaged together and renormalized to line up with the SBP of the target at low separation distances. This procedure was carried out for each target and the resulting plots inspected by eye to look for significant excess flux in the target's SBP. 
Table 1

Observation Details and Photometry

\begin{tabular}{lcccccrrr}
\hline \hline Object & UT Date & $B, V, R n_{\exp }{ }^{\mathrm{a}}$ & $B, V, R t_{\exp }(\mathrm{s})^{\mathrm{a}}$ & $r(\mathrm{px})^{\mathrm{b}}$ & $R_{h}(\mathrm{au})^{\mathrm{c}}$ & $R$ & $B-V$ \\
\hline 83982 Crantor & 2017 Aug 21 & $15,4,4$ & $1500,480,400$ & 4.5 & 18.46 & $21.20 \pm 0.03$ & $1.02 \pm 0.08$ & $0.78 \pm 0.06$ \\
121725 Aphidas & 2017 Feb 15 & $15,7,7$ & $2250,1140,720$ & 3.0 & 25.87 & $22.59 \pm 0.06$ & $1.02 \pm 0.15$ & $0.63 \pm 0.10$ \\
250112 2002 KY14 & 2017 Feb 16 & $0,1,3$ & $0,120,600$ & 3.5 & 11.91 & $20.39 \pm 0.01$ & $\cdots$ & $0.67 \pm 0.03$ \\
281371 2008 FC76 & 2017 Feb 15 & $5,4,2$ & $180,105,70$ & 3.0 & 10.65 & $19.77 \pm 0.02$ & $0.93 \pm 0.04$ & $0.65 \pm 0.03$ \\
& 2017 Feb 16 & $0,1,3$ & $0,120,420$ & 3.0 & 10.65 & $19.72 \pm 0.01$ & $\cdots$ & $0.69 \pm 0.03$ \\
167P/CINEOS & 2017 Feb 15 & $9,5,15$ & $880,150,1200$ & 3.0 & 17.15 & $22.13 \pm 0.03$ & $0.92 \pm 0.15$ & $0.43 \pm 0.12$ \\
& 2017 Aug 21 & $20,8,6$ & $3000,960,720$ & 3.5 & 17.35 & $22.17 \pm 0.03$ & $0.80 \pm 0.06$ & $0.51 \pm 0.06$ \\
174P/Echeclus & 2017 Aug 21 & $2,1,4$ & $280,120,240$ & 4.5 & 7.03 & $18.31 \pm 0.01$ & $0.93 \pm 0.01$ & $0.48 \pm 0.01$ \\
P/2008 CL94 & 2017 Aug 21 & $2,2,2$ & $200,120,120$ & 4.5 & 6.33 & $20.27 \pm 0.02$ & $0.89 \pm 0.04$ & $0.47 \pm 0.03$ \\
P/2011 S1 & 2017 Feb 16 & $0,0,4$ & $0,0,780$ & 5.5 & 7.36 & $21.88 \pm 0.05$ & $\cdots$ \\
C/2012 Q1 & 2017 Aug 21 & $8,4,4$ & $960,320,480$ & 4.5 & 12.79 & $22.21 \pm 0.04$ & $1.04 \pm 0.14$ & $0.58 \pm 0.10$ \\
\hline
\end{tabular}

Notes.

a Number of individual exposures and total exposure time in the $B, V$, and $R$ filters, respectively.

b Radius of aperture used for photometric extraction. The pixel scale is 0.363 .

${ }^{\mathrm{c}}$ Heliocentric distance at the time of observation.

\section{Results}

Across the three nights of observation, we obtained highquality photometry of nine Centaurs. Five of the targets are known active Centaurs-167P/CINEOS, 174P/Echeclus, P/ 2008 CL94, P/2011 S1, and C/2012 Q1; the remaining four are VR Centaurs-83982 Crantor, 121725 Aphidas, 250112 $2002 \mathrm{KY} 14$, and 2813712008 FC76. The calculated apparent $R$ magnitudes and $B-V$ and $V-R$ colors are listed in Table 1 along with the heliocentric distance of each object at the time of observation. The objects $167 \mathrm{P}$ and 281371 were observed on two nights, and the individual color measurements are statistically consistent to within $1 \sigma$.

We calculated both $B-V$ and $V-R$ colors for seven objects: 83982, 121725, 281371, 167P, 174P, P/2008 CL94, and C/2012 Q1. Their colors are plotted in Figure 1. We also include all other Centaur colors compiled from the published literature (Jewitt 2009, 2015; Mazzotta Epifani et al. 2014, 2018; Peixinho et al. 2015; Tegler et al. 2016). For an object with multiple observations, we take the most precise published color measurement. Some active Centaurs have photometry from multiple apertures listed; in those cases, the colors from the smallest aperture are shown, because they are most reflective of the color of the nucleus. Black dots indicate inactive Centaurs, while blue squares denote known active Centaurs not included in our observational sample. Our color measurements of active and inactive Centaurs are plotted with red triangles and green circles, respectively. Orange dashed lines link our color measurements with the literature valuesall objects have color values that are consistent with previously published values to within $2 \sigma$, with most lying well within $1 \sigma$.

From the plot, it is evident that the color distribution of inactive Centaurs is strongly bimodal, as has been pointed out by many authors (e.g., Peixinho et al. 2003, 2015; Jewitt 2015; Tegler et al. 2016; Romanishin \& Tegler 2018), dividing the population into the gray and red Centaur subpopulations. The inactive red Centaurs in our target list-83982, 121725, $281371-$ were measured to have $B-V$ and $V-R$ colors characteristic of the red subpopulation. For 250112, only the $V-R$ color was measured; it is consistent with the $V-R$ colors of the other red Centaurs as well as literature values.

Turning to the active Centaurs, no bimodality is evident. Most of these objects, including the four dormant active

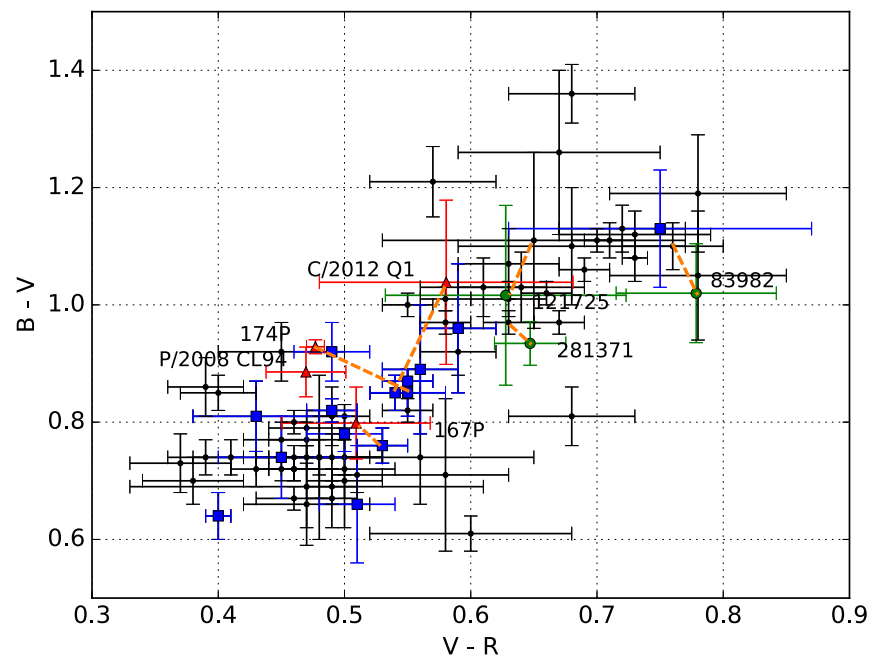

Figure 1. Color-color plot of Centaurs. Colors from our photometric observations of active and inactive Centaurs are indicated by red triangles and green circles, respectively, and are labeled by their designations. The colors of inactive Centaurs from the literature (Jewitt 2015; Peixinho et al. 2015; Tegler et al. 2016) are indicated by black dots, while previously published colors of active Centaurs (Jewitt 2009, 2015; Mazzotta Epifani et al. 2014, 2018; Peixinho et al. 2015; Tegler et al. 2016) are indicated by blue squares. For 167P, only the color measured from our 2017 August 21 observations is shown in red. Orange dashed lines link our color values with the most precise published values in the literature for the same objects, when available. The bimodality of the inactive Centaur color distribution is discernible. Active Centaurs with published colors in the literature are largely consistent with the less red of the two subpopulations, i.e., gray Centaurs, with the marked exception of the recently characterized red active Centaur 523676. The known active Centaurs in our sample have $B-V$ and $V-R$ colors that are consistent with the gray Centaurs. The inactive Centaurs in our sample belong to the red Centaur subpopulation.

Centaurs in our observational sample, have colors consistent with the gray Centaur subpopulation, while a few have colors that lie intermediate between the two color modes in the color distribution of inactive Centaurs. A notable outlier is 523676 2013 UL10, which has measured colors that lie solidly within the red Centaur subpopulation (Mazzotta Epifani et al. 2018). We note that our measurement of $\mathrm{C} / 2012$ Q1 is highly uncertain, but statistically consistent with the previous measurement by Jewitt (2015), which makes the object consistent with the gray Centaur subpopulation. 

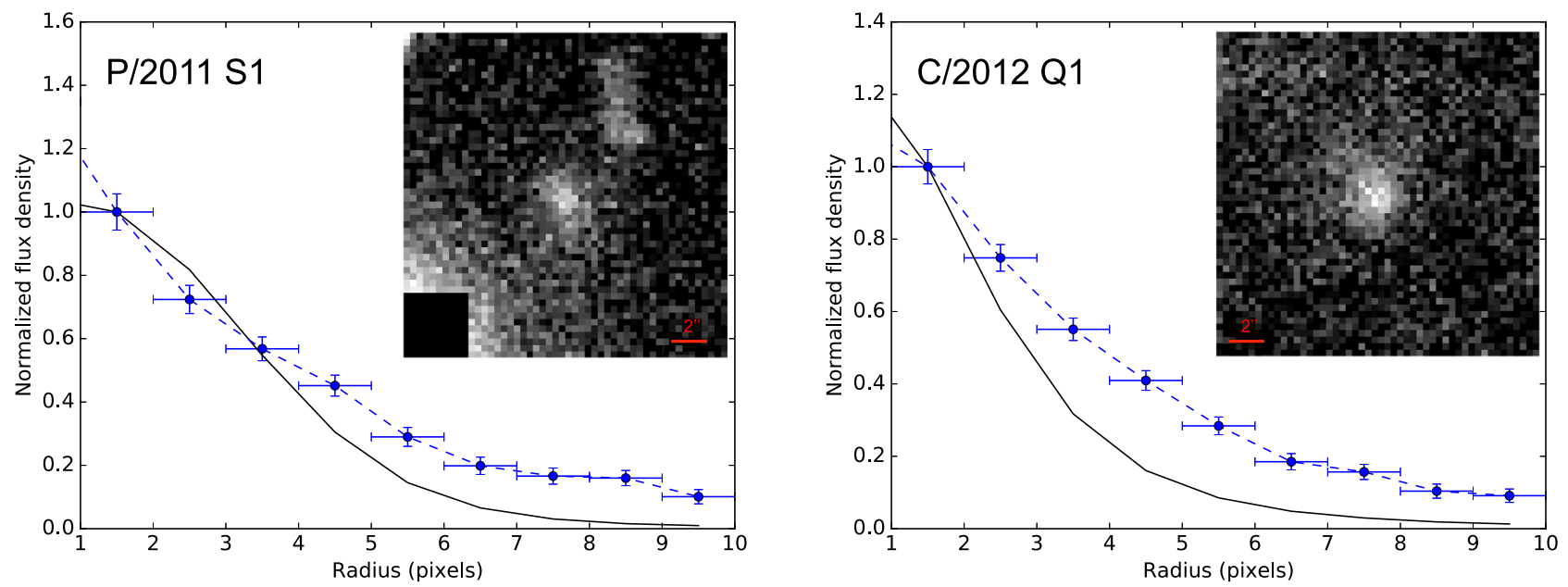

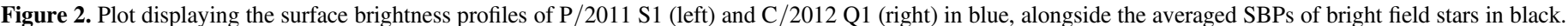

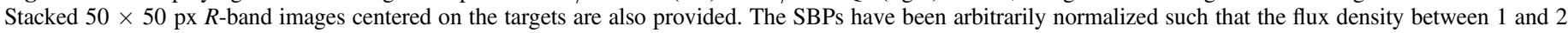

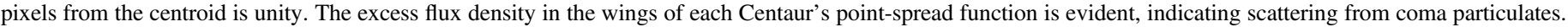

From careful inspection of both stacked images and the derived SBPs for each target, we detected activity around P/ 2011 S1 and C/2012 Q1 at the time of our observations. The averaged SBPs of these two targets are plotted alongside the averaged SBPs of field stars in Figure 2. Coadded $R$-band images, centered on the targets, are also provided. We have arbitrarily normalized all SBPs to unity between 1 and 2 pixels from the centroid. From the figure, it is evident that the SBPs of the two active Centaurs show significant deviation from the point-source SBPs, indicating reflection off coma material.

The other active Centaurs in our sample-167P, 174P, and P/2008 CL94-have SBPs that are consistent with a point source and thus were dormant at the time of our observations. It follows that their measured colors in Table 1 can be interpreted as the uncontaminated colors of their nuclei. For 167P and P/ 2008 CL94, our observations provided the first measured colors during a period of inactivity. None of the inactive Centaurs in our sample showed evidence for a coma.

In order to study the activity level of our Centaur targets in more detail and place constraints on the coma brightnesses and dust production rates, we carried out an analogous analysis to the ones presented in several previous publications on active Centaurs (e.g., Jewitt 2009; Mazzotta Epifani et al. $2011,2014)$. The magnitude of the coma in the $R$-band is calculated using the following expression:

$$
m_{c}=-2.5 \log _{10}\left\{\eta \times\left[f\left(r_{2}\right)-f\left(r_{1}\right)\right]\right\}+m_{0},
$$

where $m_{0}$ is the photometric zero-point computed from photometric calibration of each $R$-band image, and $f(r)$ is the flux contained within an aperture $r$. For the coma measurements presented in this paper, we chose $r_{1}=6 \mathrm{px}$ and $r_{2}=10$ px, corresponding to radii of 2 !' 2 and 3 ". 6 . The factor $\eta$ is a correction factor to remove the flux contribution from the wings of the nucleus' point-spread function (PSF) lying within the annulus. The value of $\eta$ was estimated by computing the fraction of each field star's PSF that is contained within the annulus and averaging. In our images, $\eta$ varied from $\sim 0.80$ to 0.98 due to the relatively large changes in seeing throughout the nights of observation (ranging from 0 ". 9 to over 2 !" 0 ).
From here, the coma cross section $\mathrm{m}^{2}$ is given by (Russell 1916)

$$
C_{c}=2.25 \times 10^{22} \frac{\pi R^{2} \Delta^{2} 10^{-0.4\left(m_{c}-m_{\odot}\right)}}{p_{R} \Phi(\alpha)},
$$

where $R$ and $\Delta$ are the heliocentric and geocentric distances (in astronomical units), and $m_{\odot}=-27.15$ is the $R$-band apparent magnitude of the Sun. For consistency with previous studies of active Centaurs (e.g., Romanishin \& Tegler 2018) and ease of comparison with other estimates of activity in the literature (e.g., Jewitt 2009; Mazzotta Epifani et al. 2011, 2014, 2018), we set the geometric albedo $p_{R}$ to 0.1 . Similarly for consistency with previous studies, we use a generic form for the phase function $\Phi(\alpha)=10^{-0.4 \beta \alpha}$, where $\alpha$ is the phase angle in degrees, and we assume $\beta=0.1 \mathrm{mag} / \mathrm{deg}$.

The total coma mass is estimated in the same way as in the analyses of Jewitt (2009) and Mazzotta Epifani et al. (2018): $M_{c}=\frac{4}{3} \rho \bar{a} C_{c}$, where we set the average size of the coma dust to $\bar{a}=30 \mu \mathrm{m}$ and assume $\rho=1000 \mathrm{~kg} \mathrm{~m}^{-3}$. It follows that the mass loss rate is simply the total coma mass $M_{c}$ divided by the average residence time of coma particles within the skyprojected annular region between $r_{1}$ and $r_{2}$. We assume that the dust particles follow the heliocentric distance-dependent trend in outflow velocity that was derived from observations of $\mathrm{C} /$ Hale-Bopp (Biver et al. 2002): $v(R)=550 \mathrm{~m} \mathrm{~s}^{-1} \times\left(5 \mathrm{au} / R_{h}\right)^{1 / 4}$. The residence time $\tau$ is thus

$$
\tau(R)=\frac{\Delta \times\left(r_{2}-r_{1}\right)}{550 \mathrm{~m} \mathrm{~s}^{-1}}\left(\frac{R_{h}}{5 \mathrm{au}}\right)^{1 / 4},
$$

where $r$ is in arcseconds and $\Delta$ is in meters.

The results of our calculations are listed in Table 2. For objects with no discernible activity, we derive $3 \sigma$ upper limits based on the standard deviation of background scatter. The relative background level in our observations of 121725 was too high and the object too faint to allow for even modest constraints on activity level, and we do not report the resultant upper limits.

In the following, we briefly summarize the previous observational studies of the active Centaurs in our sample. A 
Table 2

Centaur Activity

\begin{tabular}{lccc}
\hline \hline Object & $m_{c}{ }^{\mathrm{a}}$ & $M_{c}\left(10^{6} \mathrm{~kg}\right)^{\mathrm{b}}$ & $\dot{M}_{c}\left(\mathrm{~kg} \mathrm{~s}^{-1}\right)^{\mathrm{c}}$ \\
\hline 83982 Crantor & $>26.1$ & $<1.6$ & $<30$ \\
250112 2002 KY14 & $>26.4$ & $<0.3$ & $<10$ \\
281371 2008 FC76 & $>25.9$ & $<0.3$ & $<10$ \\
167P/CINEOS & $>26.2$ & $<1.5$ & $<30$ \\
& $>26.0$ & $<1.8$ & $<40$ \\
174P/Echeclus & $>25.7$ & $<0.09$ & $<7$ \\
P/2008 CL94 & $>26.1$ & $<0.03$ & $<3$ \\
P/2011 S1 & $22.2 \pm 0.1$ & $2.0 \pm 0.2$ & $140 \pm 20$ \\
C/2012 Q1 & $22.7 \pm 0.2$ & $7.5 \pm 1.2$ & $250 \pm 40$ \\
\hline
\end{tabular}

Notes.

a Coma magnitude between 6 and 10 pixels $(2$." $2-3$ ". 6$)$ from the center of the object. For Centaurs with no discernible activity, $3 \sigma$ upper limits are given.

${ }^{\mathrm{b}}$ Coma mass.

c Mass loss rate.

d These two measurements are derived from our observations on 2017 February 15 and August 21, respectively.

diagram illustrating the activity histories of these objects is shown in Figure 3.

\section{1. $167 P / C I N E O S$}

$167 \mathrm{P}$ is a distant active Centaur, coming to perihelion beyond the orbit of Saturn $(q=11.8 \mathrm{au})$. This object was observed to display a faint coma on 2004 October 10, when the object was at a heliocentric distance of $R_{h}=12.23 \mathrm{au}$ (Jewitt 2009). During that epoch, 167P had an $R$-band magnitude of $20.69 \pm 0.02$, with measured colors of $B-V=0.80 \pm 0.03$ and $V-R=0.49 \pm 0.10$. The mass loss rate was estimated to be $24 \mathrm{~kg} \mathrm{~s}^{-1}$. Since then, the object has continued on the outward branch of its orbit. Activity was detected on 2005 June 7-8 $\left(R_{h}=12.41 \mathrm{au}\right.$; Romanishin \& Tegler 2005), 2010 September $10\left(R_{h}=14.42 \mathrm{au}\right)$, and 2012 October $13\left(R_{h}=15.33 \mathrm{au}\right.$; Jewitt 2015). The measured colors within an aperture of radius 4!. 1 during 2010 September 10 were $B-V=0.80 \pm 0.04$ and $V-R=0.57 \pm 0.03$, while the colors within a larger 6." 8 aperture on 2012 October 13 were notably bluer $-B-V=0.68 \pm 0.05$ and $V-R=0.34 \pm 0.05$-indicating that the coma material appears significantly bluer than the nucleus.

Our observations of $167 \mathrm{P}$ from 2017 February 15 $\left(R_{h}=17.15 \mathrm{au}\right)$ and 2017 August $21\left(R_{h}=17.35 \mathrm{au}\right)$ revealed no discernible activity. We note, however, that even though inspection of the images and SBPs did not find visible signs of activity, given the quality of our observations and the much larger heliocentric distances of the target at the time, the $3 \sigma$ upper limits on mass loss rate $\left(30-40 \mathrm{~kg} \mathrm{~s}^{-1}\right)$ are still nominally consistent with the estimated value in Jewitt (2009). Our measured colors within apertures of 1 !" 1 and 1 !" 3 are consistent with each other and statistically consistent with those presented by Jewitt (2009) and Jewitt (2015) (for the smaller aperture) at better than the $1 \sigma$ level.

\section{2. $174 P /$ Echeclus}

$174 \mathrm{P}$ is one of the most well-studied Centaurs and has an activity history that is characterized by several distinct outbursts. Pre-discovery Spacewatch images of 174P showed that a discernible coma was present in 2000 January $(R=15.4$

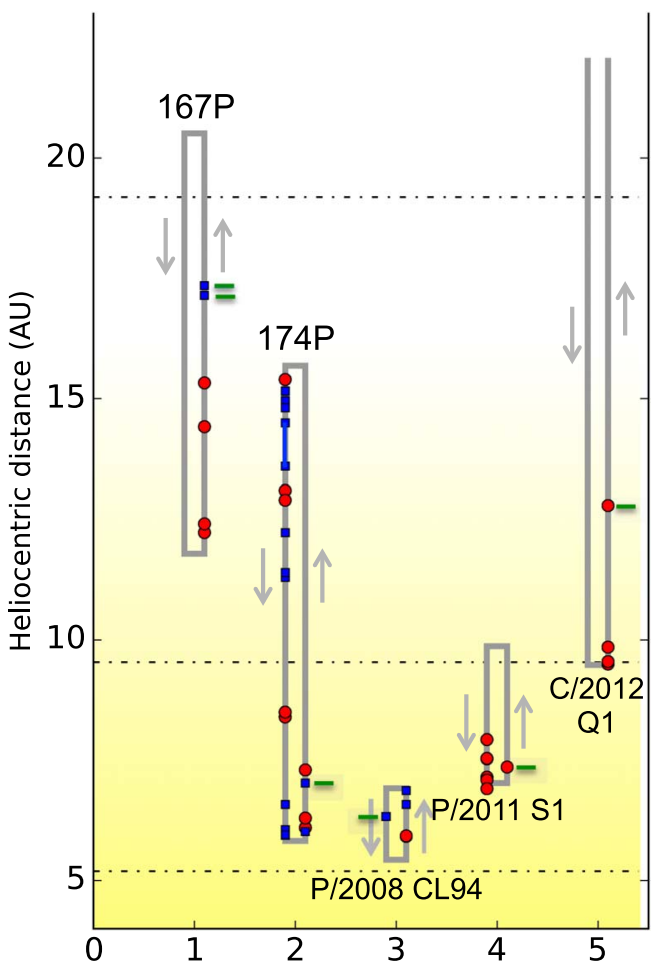

Figure 3. A summary of the published activity history for the five active Centaurs in our sample (see Sections 3.1-3.5). The gray circuits represent the objects' orbits in the counterclockwise sense. The orbital semimajor axes of Jupiter, Saturn, and Uranus are represented by horizontal lines. Red points indicate epochs when an object was observed to be active, while blue squares indicate periods of observed inactivity. Green dashes mark the data points corresponding to our observations. The yellow shaded region represents the range of heliocentric distances at which conversion of water ice from amorphous to crystalline is predicted to occur; at the largest heliocentric distances within this range, high obliquity is needed in order for this process to be an effective driver of cometary activity.

au) when the object was near aphelion (Wierzchos et al. 2017). Analysis of subsequent observations in 2001-2003 $\left(R_{h}\right.$ $=13.6-14.5 \mathrm{au}$ ) yielded a rotation period of $26.802 \pm 0.042 \mathrm{hr}$, and the first color measurements $(B-V=0.76 \pm 0.15$ and $V-R=0.51 \pm 0.09)$, but did not detect any coma (Rousselot et al. 2005). These color measurements obtained during a period of inactivity are consistent with the values we obtained from our observations. The first detected outburst occurred in late $2005\left(R_{h}=13.1 \mathrm{au}\right)$, during which 174P's brightness increased by 7 mag. (e.g., Choi \& Weissman 2006). Subsequent observations in 2006 February-April $\left(R_{h}=12.9-13.0 \mathrm{au}\right)$ revealed that the coma had a complicated and highly extended structure, discernible out to $2^{\prime}$ from the object's center (Tegler et al. 2006; Bauer et al. 2008; Rousselot 2008; Jewitt 2009). Peculiarly, the nexus of the coma was a secondary component separated from and in motion around the primary. Explanations for this unusual coma include fragmentation of the primary due to either explosive outgassing or an impact (e.g., Rousselot 2008; Fernández 2009). Multiband photometry by Bauer et al. (2008) produced color measurements of $B-V=0.77 \pm 0.06$, $V-R=0.51 \pm 0.04$ and no significant variation in color with aperture size; in particular, comparison between colors computed for a circular aperture centered on the nucleus and for an extended wide-separation annulus showed no significant difference. Rousselot (2008) presented similar color 
measurements. Estimates of mass loss rate during this first observed outburst ranged from 100 to $400 \mathrm{~kg} \mathrm{~s}^{-1}$.

Subsequent documented outburst events were observed in 2011 May-July $\left(R_{h}=8.4-8.5\right.$ au; Jaeger et al. 2011; Rousselot et al. 2016) and 2016 August-September $\left(R_{h}=6.3 \mathrm{au}\right.$; Miles et al. 2016), with the latter outburst ending in early 2016 October. Colors obtained by Rousselot et al. (2016) during the second outburst are consistent with measurements obtained during the first outburst. During the second and third outbursts, no secondary components were detected. Estimates of mass loss rate were consistently lower than values from the first outburst, being tens of $\mathrm{kg} \mathrm{s}^{-1}$. The past episodes of activity were separated by null detections of discernible coma material, when the object was at heliocentric distances of 13.6-14.5, 11.3-12.2, 5.9-6.6, and 7.0 au (Bauer et al. 2003; Rousselot et al. 2005, 2016; Lorin \& Rousselot 2007; Rousselot 2008; Choi \& Weissman 2008; Tegler et al. 2016, as well as our measurements).

Spectroscopic observations of $174 \mathrm{P}$ near its perihelion passage in 2016 May-June $\left(R_{h}=6.1 \mathrm{au}\right)$, just prior to the third documented outburst, yielded a $3.6 \sigma$ detection of $\mathrm{CO}$ emission, indicating an active gas production rate of $(7.7 \pm 3.3) \times 10^{26} \mathrm{~mol} \mathrm{~s}^{-1} \quad$ (Wierzchos et al. 2017). This makes $174 \mathrm{P}$ only the third Centaur with detected $\mathrm{CO}$, after the extremely active 29P/Schwassmann-Wachmann 1 and 2060 Chiron (see Womack et al. 2017, and references therein). An independent set of spectroscopic observations before (2014 August $3 ; R_{h}=5.95 \mathrm{au}$ ) and after (2016 October 7-8; $R_{h}=6.34 \mathrm{au}$ ) this third outburst found no significant changes to the spectral gradient from pre- to post-outburst (Seccull et al.

2019). Notably, analysis of the coma spectra yielded a blue spectral gradient of $(-7.7 \% \pm 0.6 \%) / 1000 \AA$, consistent with coeval annular aperture photometry that indicated coma colors that were significantly bluer than the near-nucleus measurements.

Most recently, a fourth large outburst was detected by amateur astronomers in 2017 December, ${ }^{5}$ when the object was $7.3 \mathrm{au}$ from the Sun. No further observations have been documented as of the writing of this paper.

\section{3. $P / 2008$ CL94}

The orbit of $\mathrm{P} / 2008$ CL94 has perihelion and aphelion distances of $q=5.43 \mathrm{au}$ and $Q=6.91 \mathrm{au}$. This object has been alternatively classified as a Jupiter-family comet (JFC) or a Centaur-JFC transition object (e.g., Horner et al. 2003; Kulyk et al. 2016). Activity was observed on 2009 March 17-18 $\left(R_{h}=5.92 \mathrm{au}\right.$; Scotti et al. 2009). Photometric and spectroscopic observations on 2009 April $1\left(R_{h}=5.93 \mathrm{au}\right)$ revealed cometary activity, an $R$-band magnitude of $19.64 \pm 0.01$, and a featureless visible spectrum with a red continuum slope of $S=(2.0 \% \pm 0.4 \%) / 1000 \AA$ (Kulyk et al. 2016). Later observations on 2011 July $7\left(R_{h}=6.58 \mathrm{au}\right)$ and 2013 May 30 $\left(R_{h}=6.87 \mathrm{au}\right.$; Kulyk et al. 2016) yielded a non-detection of a coma. Our observations on 2017 August 21 likewise did not show signs of coma material and provided the first color measurements of this object during a cessation of activity, revealing a relatively neutral surface color consistent with gray Centaurs.

\footnotetext{
http://britastro.org/node/11988
}

\section{4. $P / 2011 S 1$}

P/2011 S1 was discovered on 2011 September 18 as an active Centaur with a relatively low-eccentricity $(e=0.2)$ orbit: $q=6.9 \mathrm{au}$ and $Q=10.4 \mathrm{au}$. The discovery images were obtained when the object was 7.53 au from the Sun (Gibbs et al. 2011). A series of serendipitous observations of $P / 2011$ S1 were collected by the Pan-STARRS survey. Thirty observations, each comprising several stacked individual exposures, were made from 2010 September $29\left(R_{h}=7.93\right.$ $\mathrm{au})$ to 2012 November $4\left(R_{h}=7.15 \mathrm{au}\right)$ along the inward branch of the object's orbit (Lin et al. 2014). P/2011 S1 was active throughout these observations, though the level of the measured activity varied significantly-from 40 to $150 \mathrm{~kg} \mathrm{~s}^{-1}$, given similar assumptions for grain size and coma expansion velocities to the ones we make in this work. Subsequently, published observations from 2011 September $5\left(R_{h}=7.54 \mathrm{au}\right)$ and 2013 February $7\left(R_{h}=7.09 \mathrm{au}\right)$ likewise revealed activity, as well as a color measurement of $V-R=0.51 \pm 0.12-$ consistent with the gray Centaur subpopulation (Kulyk et al. 2016). The most recent observation previous to ours of this object took place on 2014 February $26\left(R_{h}=6.91 \mathrm{au}\right)$ and yielded the color measurements $B-V=0.96 \pm 0.11$ and $V-R=0.59 \pm 0.03$ (Jewitt 2015).

From our observations on 2017 February $16\left(R_{h}=7.36 \mathrm{au}\right)$, after the object passed perihelion, we computed a similar estimate for the activity level: $140 \pm 20 \mathrm{~kg} \mathrm{~s}^{-1}$. When accounting for the relatively small size of its nucleus $(\sim 7 \mathrm{~km}$ in diameter; Lin et al. 2014), P/2011 S1 is one of the most active Centaurs known, comparable to 29P/SchwassmannWachmann 1, 174P, and P/LG (also known as P/2010 TO20). A notable characteristic of $\mathrm{P} / 2011 \mathrm{~S} 1$ revealed by annular aperture photometry is that its coma appears to be somewhat redder than the near-nucleus region (Lin et al. 2014; Kulyk et al. 2016), which is opposite to the trend observed for $167 \mathrm{P}$ and 174P (third outburst only).

\section{5. $C / 2012 Q 1$}

C/2012 Q1 has a high-eccentricity orbit $(e=0.64)$ and a perihelion distance of $q=9.48$ au; with an orbital semimajor axis of $a=26.15 \mathrm{au}$, this object is the second most distant active Centaur known. C/2012 Q1 was discovered in images obtained from 2012 August 28 to 2012 September $4\left(R_{h}=9.5\right.$ $\mathrm{au}$ ), when a coma was reported (Miller et al. 2012). Our observations on 2017 August $21\left(R_{h}=12.79\right.$ au) likewise showed an extended coma (see Figure 2), from which we computed a comparatively high mass loss rate of $250 \pm 40 \mathrm{~kg} \mathrm{~s}^{-1}$.

A previous set of observations by Jewitt (2015) on 2012 October 13-14 $\left(R_{h}=9.55 \mathrm{au}\right)$ and 2013 October $1\left(R_{h}=9.85\right.$ $\mathrm{au}$ ) detected activity and produced color measurements at various aperture sizes. Figure 1 shows the color measurements for the smallest 2 !"7 diameter aperture $-B-V=0.85 \pm 0.03$ and $V-R=0.54 \pm 0.02$. While the $B-V$ color measurement derived from our observations is redder, our value is formally consistent with the previous value at $1.3 \sigma$, given the much larger uncertainties on our measurement. For the 5!"4 diameter aperture from the 2013 October 1 observation by Jewitt (2015), which corresponds to a spatial extent most comparable to the area around the nucleus subtended by our optimal aperture, the listed color values are $B-V=0.94 \pm 0.03$ and $V-R=0.51 \pm 0.02$; this $B-V$ 
value is more consistent with our measurement (at better than $1 \sigma)$. Jewitt (2015) carried out a multi-aperture analysis of this object and found no significant variation in color with angular radius. We conducted a similar analysis and likewise did not find any color trend, albeit with significantly larger uncertainties.

We can derive an upper limit on the nucleus size from aperture photometry of the near-nucleus region. The cross section $\mathrm{m}^{2}$ of the nucleus is given by an expression analogous to Equation (2):

$$
C_{n} \equiv \pi\left(\frac{D}{2}\right)^{2}=2.25 \times 10^{22} \frac{\pi R^{2} \Delta^{2} 10^{-0.4\left(m-m_{\odot}\right)}}{p_{R} \Phi(\alpha)},
$$

where $D$ is the diameter of the nucleus, and we set $m$ to be the $R$-band apparent magnitude of the object listed in Table 1. Using the same assumptions as before for albedo $p_{R}$ and phase function $\Phi(\alpha)$, we obtain an upper limit of $D=24 \mathrm{~km}$.

\section{Discussion}

We now discuss the implications of our results for the current understanding of Centaur activity and colors.

\subsection{Centaur Activity}

The cause of Centaur activity has been addressed in many previous works in the context of both observations and modeling. The range of heliocentric distances at which activity has been observed on these objects extends beyond the range at which water ice is unstable to sublimation, and even at the closest heliocentric distances where active Centaurs approach perihelion, the mass loss rate from sublimation of water ice is too low to account for the levels of observed activity (e.g., Jewitt 2009). Meanwhile, other ices abundant in the bulk composition of small bodies in the outer solar system, such as $\mathrm{CO}$ and $\mathrm{CO}_{2}$, are far too volatile and would sublimate readily throughout the giant-planet region if exposed at or near the surface, inconsistent with the lack of reported activity beyond $\sim 15$ au.

The leading hypothesis for the trigger of Centaur activity is the crystallization of amorphous ice (see discussion in Jewitt 2015, and references therein). Simple calculations of energy balance (Jewitt 2009) and more sophisticated thermal modeling with heat transport (Guilbert-Lepoutre 2012) have shown that complete or partial crystallization occurs on timescales comparable to the orbital stability lifetime of Centaurs at heliocentric distances smaller than $\sim 16 \mathrm{au}$. The latter study also demonstrates the effect of obliquity on the crystallization, with activity at the farthest distances requiring very high obliquity. The highly porous nature of amorphous ice can lead to trapped volatile gases such as $\mathrm{CO}$ and $\mathrm{CO}_{2}$ upon condensation from the vapor phase during planetesimal formation. When Centaurs are scattered inward from the Kuiper Belt region and attain sufficiently high surface temperatures to allow for crystallization to proceed, the trapped gases are released, expelling dust through gas drag in the process.

Our observations, along with the body of previously published results, are nominally consistent with this picture of Centaur activity (see Figure 3). The two Centaurs for which we detected activity were at 7.36 and 12.79 au from the Sun at the time of observation-within the range predicted from theory and modeling of activity driven by crystallization of amorphous ice. In particular, the very distant activity of $\mathrm{C} /$ 2012 Q1 - one of the most distant instances hitherto observed - can be explained by crystallization, provided that the object has a substantial obliquity. Previous modeling of 174P's coma morphology following its first distant outburst $\left(R_{h} \sim 13 \mathrm{au}\right)$ also indicated high obliquity (Rousselot et al. 2016). Another notable observation is the cessation of discernible activity on 167P during the outbound branch of its orbit. As illustrated in the figure, this object was last reported to be active on 2012 October 13 at a heliocentric distance of 15.33 au (Jewitt 2015). During our observations on both 2017 February 15 and August 21 , when the object was at heliocentric distances of more than $17 \mathrm{au}$, we did not detect any discernible activity. Together, these observations suggest that the activity on $167 \mathrm{P}$ ceased as the surface cooled to below the temperature at which crystallization occurs.

While the model of crystallization of amorphous ice provides an explanation for Centaur activity in general, this process alone does not account for the trends in observed activity. The documented activity history of Centaurs demonstrates that the activity on at least some objects appears to be sporadic and highly variable, even when the objects are situated within the heliocentric range for which the crystallization process is expected to be active. Besides 174P, P/2008 CL94 is an illustrative example: although this object has a close and relatively circular orbit, it has been observed to become dormant, including during our observations. We also recall the significant variations in activity level previously observed on P/2011 S1 (see Section 3.4; Lin et al. 2014).

We provide a few speculative explanations for the sporadic nature of Centaur activity within the framework of the current amorphous-to-crystalline water ice model. First, there may be significant inhomogeneities in the relative amount of trapped volatile gases in the amorphous ice matrix. Since the visible activity is driven by gas production and the associated expulsion of dust particles, if there are variations in the amount of trapped gases, either across the surface of the object or with depth, then the mass loss rate would modulate with time as the crystallization front proceeds from the surface down to the lower layers of the regolith. This explanation is also feasible for possible patches of bulk $\mathrm{CO}$ ice in the subsurface that may become accessible to drive activity as the crystallization front reaches those depths (e.g., Wierzchos et al. 2017).

A second related possibility involves inhomogeneities in the physical properties of the non-volatile regolith component. The range of latitudes for which the surface temperature rises above the crystallization temperature varies throughout a Centaur's orbit. If there are local variations in the porosity or thermal inertia of the surface regolith, which affects both heat transfer and gas permeability to the surface, then the level of activity may change as the depth and surficial extent of the active regions change. Detailed modeling of the coma morphology of both $29 \mathrm{P}$ and $174 \mathrm{P}$ during its first outburst indicated the presence of distinct localized regions of activity (Miles et al. 2016; Rousselot et al. 2016), which suggests that only small regions of the surface had the particular physical and chemical conditions necessary for outgassing.

Lastly, the consequences of activity itself may produce a sporadic temporal trend. When a Centaur is active, some of the expelled dust settles back down and blankets the surface with fine particulate matter. With time, a crust of fallback debris forms on the surface, which may severely reduce the 
permeability from the subsurface (e.g., Coradini et al. 2008). As the thermal wave continues to penetrate the surface and the crystallization front proceeds, the released volatile gas molecules are unable to escape to the surface, and as a result, pressure builds underneath the surficial crust until explosive outgassing occurs, breaking through the surface layers and producing a strong outburst in activity. Such a scenario has been invoked to explain, for example, the periodic nature of activity outbursts on $29 \mathrm{P}$ and the distinct outbursts on $174 \mathrm{P}$ (e.g., Miles et al. 2016; Wierzchos et al. 2017; Wesołowski \& Gronkowski 2018).

Stepping back to assess the broader picture, it is important to note that while the crystallization of amorphous ice is generally consistent with the body of previous observations, no incontrovertible evidence of amorphous ice has hitherto been obtained for Centaurs, or among the population of KBOs from which Centaurs are sourced. Likewise, observational studies of both short- and long-period comets have not yielded any positive detections. In light of the absence of concrete spectroscopic evidence for the existence of significant amounts of amorphous ice on the surfaces of Centaurs and KBOs, the question of the cause of Centaur activity remains open.

\subsection{Centaur Colors}

The issue of the discrepant color distributions of active and inactive Centaurs was first pointed out in Jewitt (2009). As described in Section 3 and illustrated in Figure 1, while the color distribution of inactive Centaurs displays a robust bimodality, the color distribution of active Centaurs is not discernibly bimodal, with a general lack of the redder colors characteristic of the red Centaur subpopulation. The recent photometric observations of 523676 (Mazzotta Epifani et al. 2018) revealed the first red active Centaur. In light of this, we revisit the subject of Centaur colors.

Since we are concerned with the surface color of the nuclei when addressing the color distribution of active Centaurs, the first question is whether the measurements of active Centaurs are affected by the presence of coma material. Several earlier works raised the possibility of a blueward color bias from coma particulates affecting the measured photometry of active Centaurs through non-geometric scattering off optically small dust grains (e.g., Jewitt 2009). However, multi-aperture photometry of active Centaurs as well as JFCs (e.g., Bauer et al. 2008; Rousselot 2008; Mazzotta Epifani et al. 2014; Jewitt 2015, and our analysis of C/2012 Q1 in Section 3.5) has demonstrated that while systematic trends in color with increasing angular radius do exist for some objects, these variations tend to be small, typically a few $\times 0.01 \mathrm{mag}$, which is significantly smaller than the characteristic difference in colors between gray and red Centaurs. Moreover, these trends in spectral gradient can be both positive and negative, and in most cases, the observations are statistically consistent with no variation with angular radius, given the measurement uncertainties.

There are exceptions documented in the literature, e.g., 167P (Jewitt 2015), 174P (third outburst only; Seccull et al. 2019), and 523676 (Mazzotta Epifani et al. 2018), for which comparative photometry of the near-nucleus region and pure coma revealed comae that were significantly bluer than the nucleus. Nevertheless, looking at the bigger picture, it does not appear that geometric effects impart any significant or systematic bias to the measured colors of Centaurs during periods of activity. This conclusion is strongly supported by measurements of several dormant active Centaurs (e.g., Bauer et al. 2003; Kulyk et al. 2016; Seccull et al. 2019), including the ones described in this paper for $167 \mathrm{P}, 174 \mathrm{P}$, and $\mathrm{C} / 2008$ CL94. Comparisons of the colors obtained during activity and dormancy show that the values are generally consistent. Furthermore, Jewitt (2015) demonstrated that while optically small particles are abundant in the coma material, the scattering cross section is still predicted to be dominated by larger particles, which suggests that blue scattering is not generally expected.

Having argued against systematic bias in the measured colors of active Centaurs due to blue scattering off small coma particles, we now proceed to a statistical analysis of the Centaur color distribution. The left panel in Figure 4 shows the $B-R$ color distribution for active and inactive Centaurs. Carrying out a non-parametric two-sample Kolmogorov-Smirnov (KS) test on these two color distributions, we obtain a $p$-value of 0.13 , indicating that the chance of the two distributions being drawn from the same underlying distribution is $13 \%$. For a Gaussian distribution, this value corresponds to a roughly $1.5 \sigma$ difference, which is lower than the roughly $2 \sigma$ difference reported by Jewitt (2015) prior to the discovery of the red active Centaur 523676.

In the previous subsection, we described the prevailing hypothesis that ascribes the cause of Centaur activity to the crystallization of amorphous ice. Since this process is only effective at relatively small heliocentric distances, a more diagnostic statistical analysis is to compare the color distributions of active and inactive Centaurs with orbits that would allow for activity to occur. In the right panel of Figure 4, we plot the color distributions of active and inactive Centaurs, limiting the samples to objects with perihelion distances less than $10 \mathrm{au}$. This arbitrary cutoff includes all active Centaurs except 167P. Varying the cutoff distance by several au does not significantly affect the results. Running the KS test on these low-perihelion color distributions returns $p=0.42$, which corresponds to a $0.8 \sigma$ difference. Therefore, we conclude that the color distribution of active Centaurs does not differ from that of inactive Centaurs with similar orbits in a statistically significant way.

The newly demonstrated statistical consistency of the color distributions of active and inactive Centaurs strongly hinges upon the addition of the red Centaur 523676 to the sample of active objects. It has been proposed that blanketing of the strongly irradiated regolith by unirradiated fallback material from the ejected coma could be responsible for quickly converting initially red Centaurs to gray objects upon the onset of activity (e.g., Jewitt 2009, 2015). Mazzotta Epifani et al. (2018) report that the coma of 523676 is significantly more neutral than the near-nucleus region and is consistent with the color of gray Centaurs. They invoke the blanketing hypothesis in suggesting that the blanketing process on that Centaur must be incipient. Order-of-magnitude estimates for the characteristic blanketing timescale for such an object are very short, ranging from tens to hundreds of years, even given the relatively low calculated low mass loss rate of $\sim 10 \mathrm{~kg} \mathrm{~s}^{-1}$. Assuming a typical Centaur lifetime of $10^{6-7} \mathrm{yr}$, this means that 523676 must have been observed within the first $10^{-6}-10^{-4}$ fraction of its lifetime-comparable to a few orbital timescales.

The blanketing scenario predicts that all Centaurs, regardless of their primordial color, become gray once activity initiates. It 

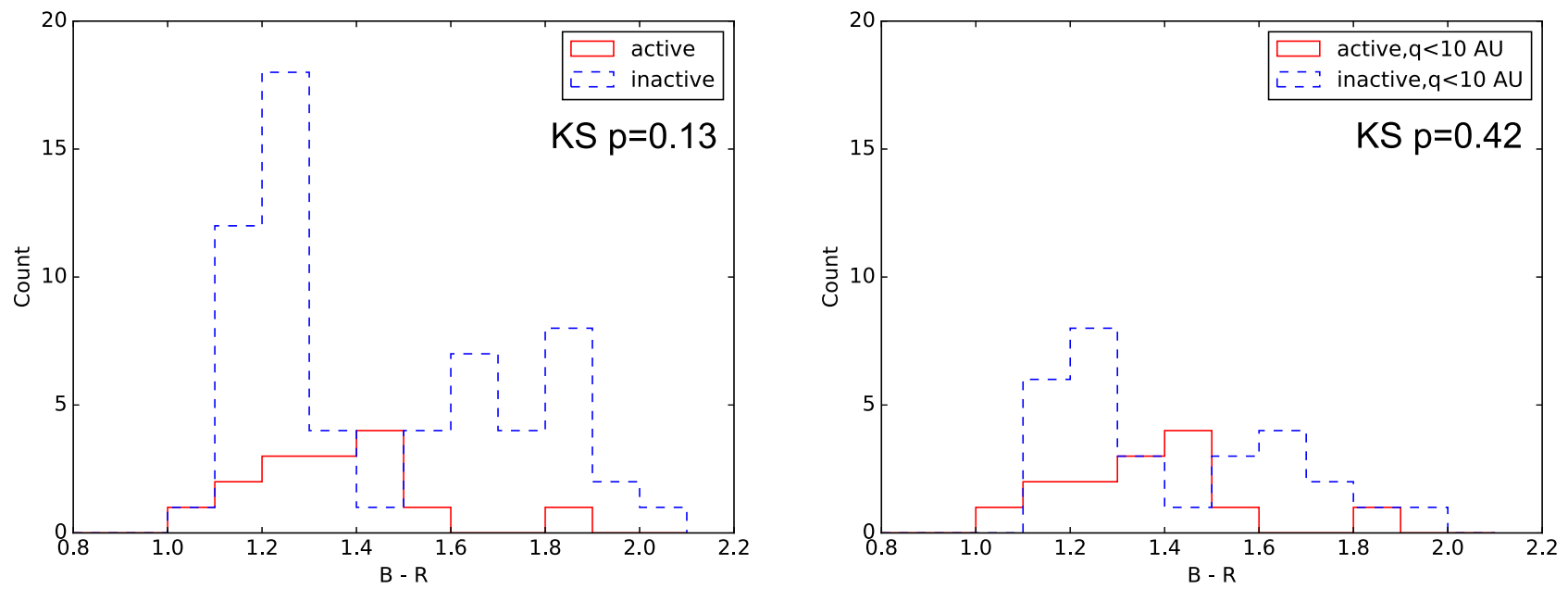

Figure 4. Left: histogram of $B-R$ colors of active and inactive Centaurs (Jewitt 2009, 2015; Mazzotta Epifani et al. 2014, 2018; Peixinho et al. 2015; Tegler et al. 2016, and this work), along with the calculated $p$-value from a two-sample Kolmogorov-Smirnov test on the two color distributions. Right: the same, but limited to objects with orbital perihelia less than 10 au.

follows that the color distribution of active Centaurs should be unimodal and therefore distinct from that of inactive Centaurs, with 523676 representing an exceptional object that was fortuitously observed almost immediately after the first onset of activity.

While we cannot rule out this scenario, we also cannot categorically rule out the possibility that geometric effects may be the explanation for the significantly more neutral coma of 523676. In other documented cases of significantly bluer comae, such as 167P and 174P (see Sections 3.1 and 3.2), there were epochs during which relatively blue coma colors were measured, as well as observations at other times that indicated no significant color difference between coma and nucleus. This suggests that the instances of relatively blue comae may not have been representative of the intrinsic color of the dust, but rather contingent upon some particular aspect(s) of the coma morphology during those times. Future observations of 523676 might likewise show a coma color that is more consistent with the red color of the nucleus.

\subsubsection{The Possible Role of Thermal Processing}

Another dimension in the discussion of Centaur colors is orbital history. In Section 4.1, we described how the observed activity history of Centaurs is consistent with crystallization of amorphous ice being the primary driver of activity. This temperature-driven process is only effective at heliocentric distances less than $\sim 16$ au (Guilbert-Lepoutre 2012). Naturally, this means that the average perihelion distance of active Centaurs is smaller than that of the overall Centaur population, as well as the inactive Centaur population, a fact that has been demonstrated by several previous works (e.g., Jewitt 2009, 2015). In particular, dynamical simulations of Centaurs have shown that, based on their current orbits, active Centaurs spend on average a significantly larger fraction of time at smaller heliocentric distances than inactive Centaurs (Melita \& Licandro 2012; Fernández et al. 2018). Notably, these same simulations also show that both inactive and active gray Centaurs have statistically closer orbits than red Centaurs.

Since the close encounters that scatter KBOs inward onto Centaur orbits are random, there is no reason why gray KBOs would be preferentially scattered onto lower-perihelion orbits than red KBOs. It follows that some secondary process must be responsible for neutralizing the surface material on red Centaurs with low-perihelion orbits, thereby producing the apparently divergent orbital distributions of gray and red Centaurs. Within the blanketing hypothesis, this discrepancy would be due to the enrichment of gray objects in the population of inactive low-perihelion Centaurs as a consequence of activity: red objects that have low perihelia become active, and the activity rapidly blankets their surfaces with unweathered, relatively neutral dust, before the object eventually becomes permanently dormant. Given the recent observation of a red active Centaur, as well as several lowperihelion inactive red Centaurs with orbits that would allow for activity (see right panel of Figure 4), we propose instead that thermal processing of the surface may be the cause of redto-gray Centaur conversion.

The scenario is as follows: gray and red KBOs are scattered into the giant-planet regions randomly, and initially the two color subpopulations are distributed evenly in the space of orbital semimajor axis and perihelion distance. Some of the Centaurs (both gray and red) that have closer-in orbits become active, though the activity does not directly affect the surface colors. Over time, objects that have closer orbits accumulate higher levels of irradiation and heating and become gray, even if activity never occurs and the objects remain inactive. Eventually, the surface color of all red Centaurs reaching a certain threshold of thermal processing is neutralized, with only the more distant red Centaurs retaining their primordial color over long timescales.

For another perspective on this hypothesis, we turn to JFCs. Like the Centaurs, JFCs are also former KBOs that have been scattered inward, but their orbits lie much closer to the Sun, with perihelion distances near or below that of Jupiter. The color distribution of JFCs differs dramatically from that of Centaurs, lacking red objects entirely (Jewitt 2015). In the context of our hypothesis, the reason for this is straightforward -all of the JFCs have undergone vigorous heating and irradiation, regardless of whether cometary activity ever occurred, and the initially red objects have rapidly neutralized at the significantly higher temperatures of the inner solar system.

The general lack of robust constraints on the detailed composition of Centaurs means that the qualitative hypothesis 
outlined above is necessarily speculative. In particular, the relevant timescale over which the thermal processing would occur is uncertain, though it could in principle be longer than the blanketing timescale for active Centaurs, thereby allowing for red active Centaurs to retain their color for longer than a few orbital periods. A discovery of another red active Centaur, for example, would strongly disfavor the blanketing explanation for the neutralization of Centaur colors, since the chances of observing two objects within the first $10-100 \mathrm{yr}$ of their activity lifetimes are astronomically small, as explained previously.

We mention in passing that models of KBOs, and by extension Centaurs, predict that the surfaces of these objects are composed of a refractory irradiation mantle from the weathering of primordial volatile ices in the outer solar system (e.g., Brown et al. 2011; Wong \& Brown 2016). Previous experimental work has shown that irradiation of volatile ices leads to a general reddening of the visible spectral gradient to values typical for KBOs and the production of complex hydrocarbons (e.g., Brunetto et al. 2006). Laboratory studies of similar high-molecular-weight hydrocarbons, such as asphaltite and kerite, as well as direct irradiation products of volatile ice mixtures, have demonstrated the neutralization of the optical color under irradiation, due to carbonization of the surface (e.g., Moroz et al. 2004; Poston et al. 2018). Complex organics similar to the ones analyzed in irradiation studies have been detected on the surface of the highly active JFC 67P/ Churyumov-Gerasimenko (e.g., Wright et al. 2015), so we consider the aforementioned hypothesis of the neutralizing effect of thermal processing to be at least plausible.

\section{Conclusion}

We have presented the results of multiband photometric observations of Centaurs: five active Centaurs-167P/ CINEOS, 174P/Echeclus, P/2008 CL94, P/2011 S1, C/ 2012 Q1-and four inactive red Centaurs-83982 Crantor, 121725 Aphidas, 2501122002 KY14, 2813712008 FC76.

1. Activity was detected on two Centaurs-P/2011 S1 and $\mathrm{C} / 2012$ Q1. Estimation of the activity level using similar assumptions to previous works in the literature yielded relatively high mass loss rates of $140 \pm 20$ and $250 \pm 40 \mathrm{~kg} \mathrm{~s}^{-1}$, respectively.

2. No discernible comae were found on the active Centaurs 167P/CINEOS, 174P/Echeclus, and P/2008 CL94 at the time of our observations. The measured $B-V$ and $V-R$ colors of these Centaurs during inactivity are consistent with the colors of the gray Centaur subpopulation and statistically identical to previously published colors obtained during periods of activity.

3. We did not detect the onset of activity on the four inactive red Centaurs in our sample.

4. The observed activity history of active Centaurs continues to be consistent with the hypothesis that crystallization of amorphous water ice is the primary trigger.

5. Comparisons of measured colors of active Centaurs obtained during periods of activity and dormancy indicate that photometric observations of active Centaurs are generally not affected by significant contamination from blue scattering off coma particulates.

6. With the discovery of the red active Centaur 523676 (Mazzotta Epifani et al. 2018), the color distributions of active Centaurs and inactive Centaurs with similar orbits are now statistically consistent to within $1 \sigma$.

7. We propose that the higher levels of thermal processing experienced by lower-perihelion Centaurs, both active and inactive, might lead to neutralization of their surface colors, resulting in the observed divergence in perihelion distance distributions between gray and red Centaurs.

I.W. is supported by a Heising-Simons Foundation 51 Pegasi b postdoctoral fellowship. A.M. thanks the Research Internship in Science and Engineering program organized by Boston University for the summer research opportunity that contributed substantially to this work. This work made use of the JPL Solar System Dynamics high-precision ephemerides through the HORIZONS system.

\section{ORCID iDs}

Ian Wong (i) https://orcid.org/0000-0001-9665-8429 Michael E. Brown (ib https://orcid.org/0000-0002-8255-0545

\section{References}

A’Hearn, M. F., Campins, H., Schleicher, D. G., \& Millins, R. L. 1989, ApJ, 347, 1155

Bauer, J. M., Choi, Y.-J., Weissman, P. R., et al. 2008, PASP, 120, 393 Bauer, J. M., Meech, K. J., Fernández, Y. R., et al. 2003, Icar, 166, 195 Biver, N., Bockelée-Morvan, D., Colom, P., et al. 2002, EM\&P, 90, 5 Brown, M. E., Schaller, E. L., \& Fraser, W. C. 2011, ApJL, 739, L60 Brunetto, R., Barucci, M. A., Dotto, E., \& Strazzulla, G. 2006, ApJ, 644, 646 Capria, M. T., Coradini, A., De Sanctis, M. C., \& Orosei, R. 2000, AJ, 119,3112

Choi, Y.-J., \& Weissman, P. 2006, BAAS, 38, 551

Choi, Y. J., \& Weissman, P. 2008, LPICo, 1405, 8355

Clark, B. E., Ziffer, J., Nesvorny, D., et al. 2010, JGR, 115, E06005

Coradini, A., Capria, M. T., de Sanctis, M. C., \& McKinnon, W. B. 2008, in The Solar System Beyond Neptune, ed. M. A. Barucci et al. (Tucson, AZ: Univ. Arizona Press), 243

Delsanti, A., Hainaut, O., Jourdeuil, E., et al. 2004, A\&A, 417, 1145

Duffard, R., Ortiz, J. L., Thirouin, A., Santos-Sanz, P., \& Morales, M. 2009, A\&A, 505, 1283

Fernández, J. A., Helal, M., \& Gallardo, T. 2018, P\&SS, 158, 6

Fernández, Y. R. 2009, P\&SS, 57, 1218

Flewelling, H. A., Magnier, E. A., Chambers, K. C., et al. 2016, arXiv:1612. 05243

Fraser, W. C., \& Brown, M. E. 2012, ApJ, 749, 33

Gibbs, A. R., Tornero, S. F., \& Williams, G. V. 2011, IAUC, 9234

Guilbert-Lepoutre, A. 2012, AJ, 144, 97

Hainaut, O. R., Boehnhardt, H., \& Protopapa, S. 2012, A\&A, 546, L115

Horner, J., Evans, N. W., Bailey, M. E., \& Asher, D. J. 2003, MNRAS, 343, 1057

Jaeger, M., Prosperi, E., Vollmann, W., et al. 2011, IAUC, 9213, 2

Jewitt, D. 2009, AJ, 137, 4296

Jewitt, D. 2015, AJ, 150, 201

Kulyk, I., Korsun, P., Rousselot, P., Afanasiev, V., \& Ivanova, O. 2016, Icar, 271,314

Lin, H. W., Chen, Y. T., Lacerda, P., et al. 2014, AJ, 147, 114

Lorin, O., \& Rousselot, P. 2007, MNRAS, 376, 881

Mazzotta Epifani, E., Dall'Ora, M., Perna, D., Palumbo, P., \& Colangeli, L. 2011, MNRAS, 415, 3097

Mazzotta Epifani, E., Dotto, E., Ieva, S., et al. 2018, A\&A, 620, A93

Mazzotta Epifani, E., Perna, D., Licandro, J., et al. 2014, A\&A, 565, A69

Melita, M. D., \& Licandro, J. 2012, A\&A, 539, A144

Miles, R. 2016, CBET, 4313

Miles, R., Faillace, G. A., Mottola, S., et al. 2016, Icar, 272, 327

Miller, P., Roche, P., Tripp, A., et al. 2012, MPEC, 2012, 2012-R08

Moroz, L., Baratta, G., Strazzulla, G., et al. 2004, Icar, 170, 214

Peixinho, N., Delsanti, A., \& Doressoundiram, A. 2015, A\&A, 577, A35

Peixinho, N., Delsanti, A., Guilbert-Lepoutre, A., Gafeira, R., \& Lacerda, P. 2012, A\&A, 546, A86

Peixinho, N., Doressoundiram, A., Delsanti, A., et al. 2003, A\&A, 410, L29

Poston, M. J., Mahjoub, A., \& Ehlmann, B. L. 2018, ApJ, 856, 124 
Romanishin, W., \& Tegler, S. C. 2005, IAUC, 8545, 1

Romanishin, W., \& Tegler, S. C. 2018, AJ, 156, 19

Rousselot, P. 2008, A\&A, 480, 543

Rousselot, P., Korsun, P., Kulyk, I., et al. 2016, MNRAS, 462, S432

Rousselot, P., Petit, J.-M., Poulet, F., \& Sergeev, A. 2005, Icar, 176, 478

Russell, H. N. 1916, ApJ, 43, 173

Schwamb, M. E., Fraser, W. C., Bannister, M. T., et al. 2019, ApJS, in press

Scotti, J. V., Bressi, T. H., Spahr, T. B., et al. 2009, MPEC, 2009, 2009-F28

Seccull, T., Fraser, W. C., Puzia, T. H., Fitzsimmons, A., \& Cupani, G. 2019 , AJ, 157, 88
Tegler, S., Consolmagno, G., \& Romanishin, W. 2006, IAUC, 8701, 1

Tegler, S. C., Romanishin, W., \& Consolmagno, G. J. J. S. 2016, AJ, 152, 210

Tiscareno, M. S., \& Malhotra, R. 2003, AJ, 126, 3122

Tonry, J. L., Stubbs, C. W., Lykke, K. R., et al. 2012, ApJ, 750, 99

Wesołowski, M., \& Gronkowski, P. 2018, EM\&P, 121, 105

Wierzchos, K., Womack, M., \& Sarid, G. 2017, AJ, 153, 230

Womack, M., Sarid, G., \& Wierzchos, K. 2017, PASP, 129, 031001

Wong, I., \& Brown, M. E. 2016, AJ, 152, 90

Wong, I., \& Brown, M. E. 2017, AJ, 153, 145

Wright, I. P., Sheridan, S., Barber, S. J., et al. 2015, Sci, 349, 020673 